\title{
Ultra-wideband Radio Fuze Time Domain Doppler Effect Research
}

\author{
Shen Lei \\ Mechatronical Engineering, \\ Beijing Institute of Technology \\ Beijing, \\ matouzi417@bit.edu.cn
}

\author{
Huang Zhonghua \\ Mechatronical Engineering, \\ Beijing Institute of Technology \\ Beijing, \\ huangzh@bit.edu.cn
}

\begin{abstract}
The Doppler effect of UWB signal and the influence on Fuze receiver output signal is quite different from the sinusoidal narrow-band signal.It is necessary to find the relationship between the frequency of fuze receiver and the projectile target approaching velocity. The article study the time domain Doppler effect of UWB fuze receiver,derivation the expression of fuze receiver input output signal,simulation the frequency relationship according to the mathematical model of UWB fuze receiver.The simulation results shows the output signal of the UWB receiver is proportional to the projectile target approaching velocity and input frequency, The output signal of the UWB receiver is inverse ratio to the width of fuze transmit pulse signal.
\end{abstract}

Keywords-Ultra-wideband fuze; Ultra-wideband signal;time domain Doppler effect; Fuze receriver;projectile target velocity

\section{INTRODUCTIONS}

Doppler effect first found by Austria physicist Doppler in 1842, the essence of Doppler effect is when there is relative motion between the transmitter and the receiver, oscillation frequency received by the receiver is different from the transmitter frequency. In the radio fuze system, the fundamental reason cause the Doppler effect is that echo signal duration changed compared with the emission signal when there is relative motion between fuze and target[1,2].

For sinusoidal narrow-band signal, the changes of echo signal duration reflects the change of signal carrier frequency. If the transmit signal frequency is $f_{0}$,Velocity of projectile target is $v_{\mathrm{r}}$, the frequency of the echo signal is as "(1)":

$$
f=f_{0}+f_{\mathrm{d}}=f_{0} \pm \frac{2 f_{0} v_{\mathrm{r}}}{c}=f_{0} \pm \frac{2 v_{\mathrm{r}}}{\lambda_{0}}
$$

$f$ is frequency of the echo signal, $f_{\mathrm{d}}$ is Doppler frequency. If projectile and target is approaching, $f_{\mathrm{d}}$ is positive value,if projectile and target is apart, $f_{\mathrm{d}}$ is negative value.
For Ultra wide band(UWB) radio fuze,the frequency band of transmit signal is wide,the "(1)" is not suit for the UWB fuze.Actually, the Doppler effect of UWB signal and the influence on Fuze receiver output signal is quite different from the sinusoidal narrow-band signal[3-5].

\section{UWB SIGNAL DOPPLER TIME SHIFT}

Assume that the receiver is located at coordinates $\mathrm{K}$, the transmit is located at coordinates $\mathrm{K}^{\prime}$,the transmitter coordinates move to the right along the $\mathrm{x}$ axis at a speed $v$ relative to the receiver coordinates.As Figure 1.shows.

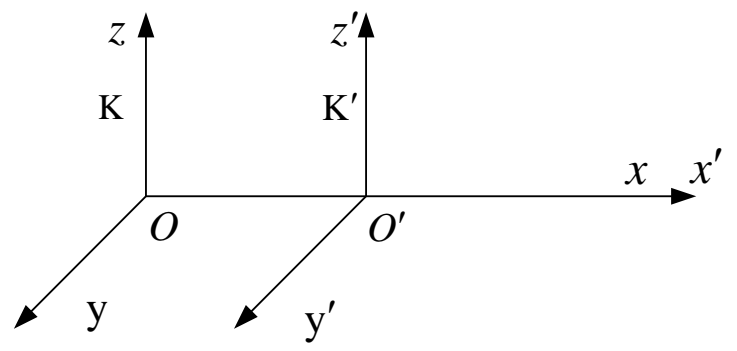

Figure 1.Doppler effect

In the transmitter discrete signal process, mark two time points $t_{1}$ and $t_{2}$ on the receiver coordinates $\mathrm{K}$,the corresponding instantaneous wave source of $t 1$ and $t 2$ is $x 1$ and $\mathrm{x} 2$,the duration of the transmitter signal(the clock according to the coordinate $\mathrm{k}$ ) is:

$$
\begin{aligned}
& \eta=t_{2}-t_{1} \\
& x_{2}=x_{1}+\eta v
\end{aligned}
$$

The starting and ending signal of the receiver $S_{1}$ and $\varsigma_{2}$ (on the coordinate $\mathrm{K}$ ) is:

$$
\begin{aligned}
& \varsigma_{1}=t_{1}+\frac{x_{1}}{c} \\
& \varsigma_{2}=t_{2}+\frac{x_{2}}{c}
\end{aligned}
$$

$c$ is velocity of electromagnetic wave.

From"(4)"and"(5)", the duration time of the receiver is: 


$$
\begin{aligned}
& \varsigma=\varsigma_{2}-\varsigma_{1}=t_{2}-t_{1}+\frac{\left(x_{2}-x_{1}\right)}{c}=t_{2}-t_{1}+\frac{\left(t_{2}-t_{1}\right) v}{c} \\
& =\eta\left(1+\frac{v}{c}\right)
\end{aligned}
$$

On the coordinate $\mathrm{K}^{\prime}$

$$
\varsigma=\eta^{\prime}\left(1+\frac{v}{c}\right)
$$

In the radio fuze system, The transmitter and receiver are located in the same projectile,the receiver signal is the target reflection signal,the duration time of signal is :

$$
\varsigma=\eta^{\prime}\left(1+\frac{2 v}{c}\right)
$$

From "(8)", when there is relative motion between fuze and target, echo signal

$$
\text { duration time changed }\left(1+\frac{2 v}{c}\right) \text { multiple compared }
$$
with the emission signal.

The Doppler effect of UWB signal is the change of pulse width and pulse repetition cycle.,the pulse width of the echo signal and the repetition cycle is[6-8]:

$$
\begin{gathered}
\Delta T^{\prime}=\Delta T+\Delta T_{\mathrm{d}}=\Delta T \pm \frac{2 \Delta T v_{\mathrm{r}}}{c} \\
T^{\prime}=T+T_{\mathrm{d}}=T \pm \frac{2 T v_{\mathrm{r}}}{c}
\end{gathered}
$$

If the target is approaching, $\Delta T_{\mathrm{d}}$ and $T_{\mathrm{d}}$ is negative,.if the target is getting far away, $\Delta T_{\mathrm{d}}$ and $T_{\mathrm{d}}$ is positive valu e.

For UWB signal, if $\Delta T$ is $1 \mathrm{~ns}, \nu_{\mathrm{r}}=100 \mathrm{~m} / \mathrm{s}$ we can calculate $\Delta T_{\mathrm{d}}=6.7 \times 10^{-7} \mathrm{~ns}$, ignore the distance between the projectile target during the single pulse propagation caused by relative motion,that is $\Delta T$ change,for point target $\Delta T^{\prime}=\Delta T$. But $\mathrm{T}$ is relative large,the distance between the projectile and target can not be ignored,so the pulse repetition period is called UWB signal Doppler effect in time domain, $T_{\mathrm{d}}$ is called Doppler shift.

Assume the projectile and target is approaching,in the lauch of ith pulse,the distance between fuze and target is

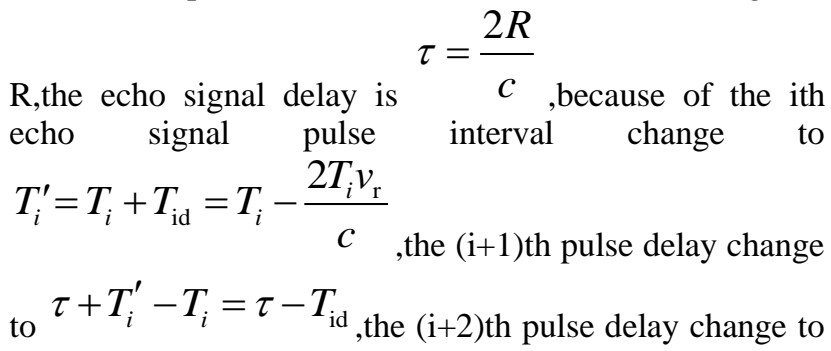

$\tau-T_{\mathrm{id}}-T_{(\mathrm{i}+1) \mathrm{d}}$,and so on,the $(\mathrm{i}+\mathrm{j})$ th pulse delay change $\tau-\sum_{m=i}^{i+j-1} T_{\mathrm{md}}$

as the Figure 2. Shows $[9,10]$.

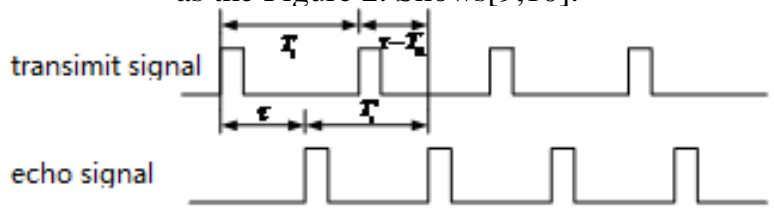

Figure 2.time domain Doppler effect

\section{INFLUENCE OF TIME DOMAIN DOPPLER EFFECT ON UWB RECEIVER OUTPUT SIGNAL}

The UWB radio fuze mainly adopts the way of sampling integral to receive.The basic principle of sampling integral as Figure 3. shows. Trigger sampling pulse generating circuit generates a pulse width is $T_{\mathrm{g}}$ 、 repetition period is $\mathrm{T}$ sampling pulse. When sampling pulse is coming the switch $\mathrm{K}$ closed,integrated circuit integral sampling to the input signal, during the sampling pulse interval,switch $\mathrm{K}$ break off,the integral circuit keep the result during $T_{\mathrm{g}}$.

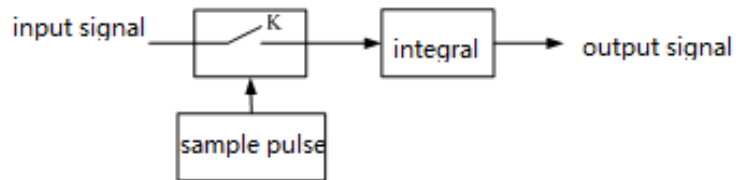

Figure 3.basic theory of sampling integral

If the trigger signal of sampling pulse is the fixed delay of transimiit signal,the sampling pulse will sample at a fixed position.The time domain Doppler effect will change the relative position of sample pulse,the echo signal through the sampling gate $T_{\mathrm{id}}=\frac{2 v_{\mathrm{r}} T_{i}}{c}=\frac{2 v_{\mathrm{r}}\left(T+X_{i}-X_{i-1}\right)}{c}$ as the Figure 4 shows.

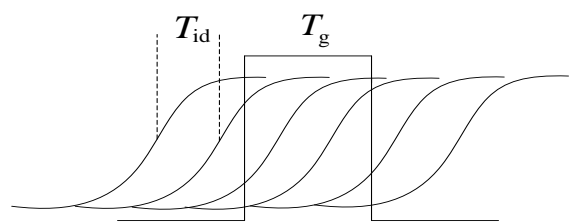

Figure 4.the relationship between echo signal and sampling gate

The time domain Doppler effect on UWB signal will lead the sample integral circuit work under the scan mode,it is mean the sample pulse sampling the single echo pulse by $T_{\text {id }}$ as the sample interval.the echo signa submerged in noise will be recovered according several periods.

From the above analysis, the echo signal through the fuze receiver sampling gate by $T_{\text {id }}$, it is mean the sample pulse sampling the single echo pulse by $T_{\text {id }}$ as the sample 
interval, $_{\mathrm{id}}=\frac{2 T_{i} v_{\mathrm{r}}}{c}$.As Figure 5. Shows.A period signal that duration time is $l$,needs $M=\frac{l}{T_{\mathrm{d}}}$ period from the sample pluse start to sample to the end,among them, $T_{\mathrm{d}}=\frac{2 T v_{\mathrm{r}}}{c}$.The receiver output signal length change to"(11)"

$$
l^{\prime} \approx M T=\frac{l T}{T_{\mathrm{d}}}=\frac{c}{2 v_{\mathrm{r}}} l
$$

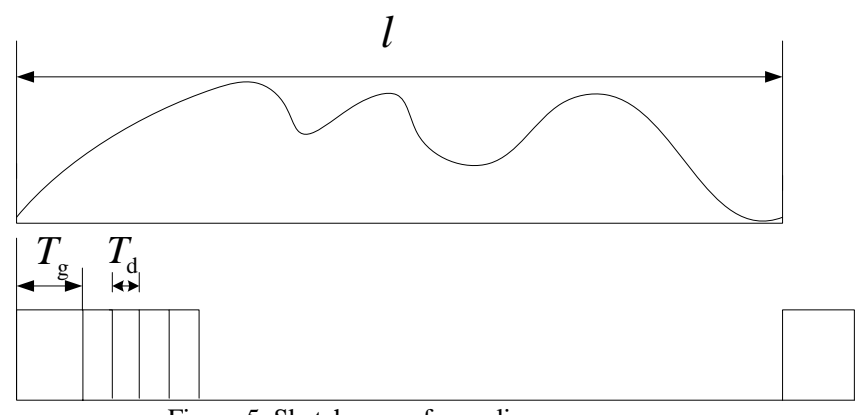

Figure 5. Sketch map of sampling

From “(11)",the signal length changed $\frac{c}{2 v_{\mathrm{r}}}$ multiple compared with the past,assume the circuit output signal waveform ont changed, the circuit output signal frequency

changed $\frac{2 v_{\mathrm{r}}}{c}$ multiple compared with the past.As the "(12)"shows:

$$
f^{\prime}=\frac{2 v_{\mathrm{r}}}{c} f
$$

$f$ Is the frequency of input signal, $f^{\prime}$ is the frequency of output signal.From "(12)",the output signal of the UWB receiver is proportional to the projectile target approaching velocity $v_{\mathrm{r}}$ and input frequency.

The above analysis is based on the invariance of the signal waveform. In actual situation, sampling pulse has a certain width, the echo signal is not constant during the sampling gate open.so the sampling output signal is an average of the sampling capacitor.Besides,the balanced sampling integral circuit can not fully recover the amplitude of input signal, the gain is different,the waveform of output signal maybe changed.The "(12)" is an approximate relationship.

\section{FUZE TIME DOMAIN DOPPLER EFFECT SIMULATION}

Simulation according to the mathematical model of UWB fuze receiver and the frequency relation of Doppler effect on the receiver input, output signal.The receiver input signal is fuze echo signal,as Figure 6.and Figure 7. shows.

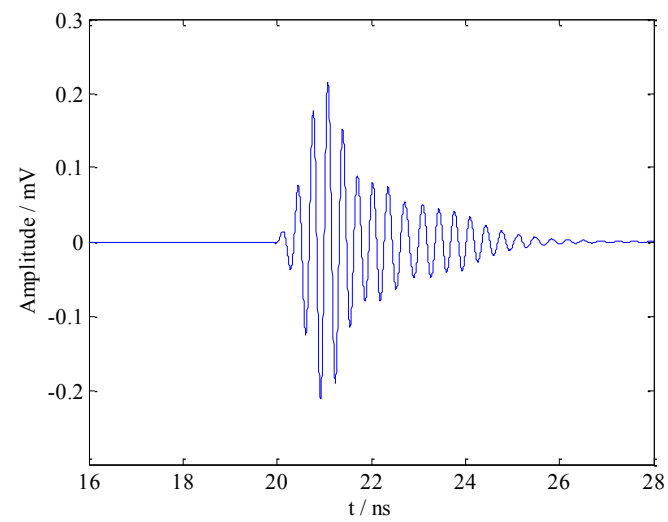

Figure 6.echo signal time domain waveform

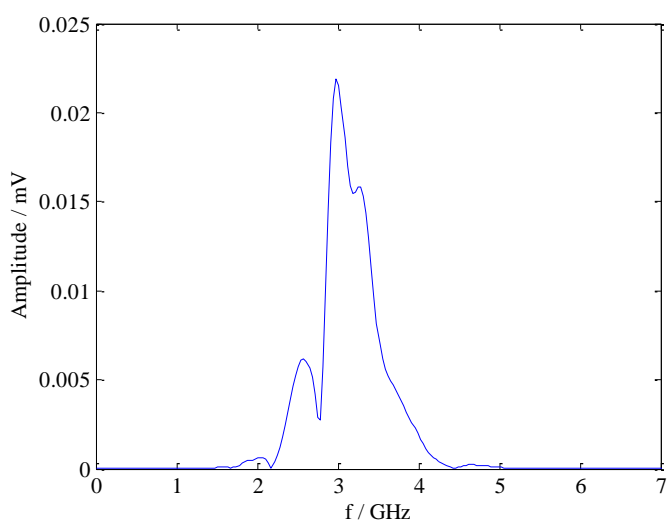

Figure 7.echo signal frequency spectrum

The velocity of projectile respectively equal to $100 \mathrm{~m} / \mathrm{s}, 200 \mathrm{~m} / \mathrm{s}, 300 \mathrm{~m} / \mathrm{s}$, the fuze receiver output signal waveform and spectrum as Figure 8.and Figure 9. shows.

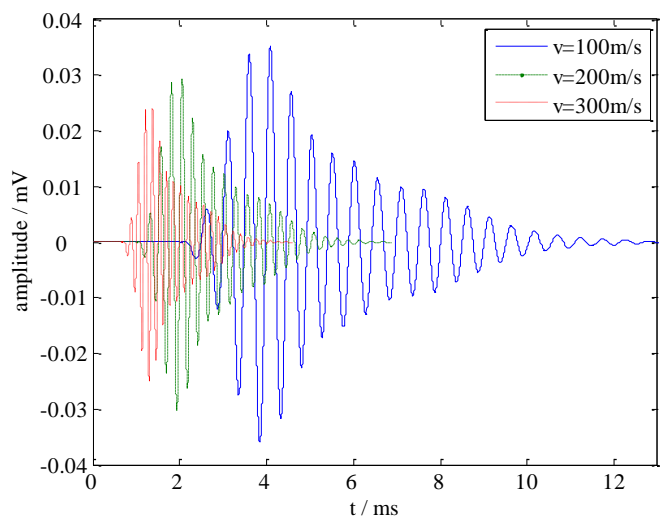

Figure 8.output signal time domain waveform 


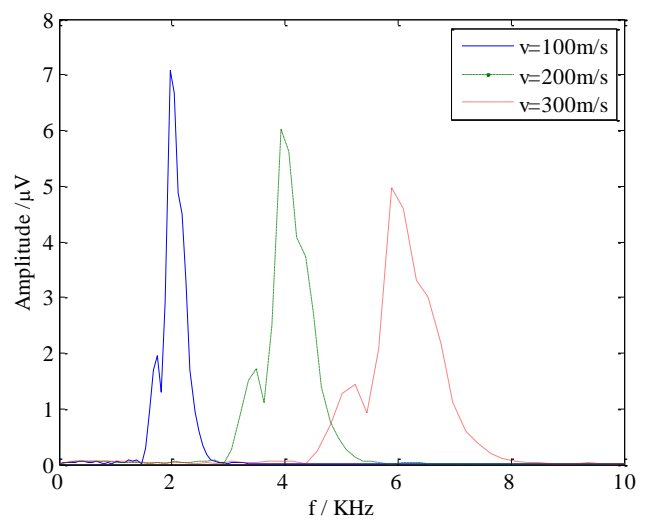

Figure 9.output signal frequency spectrum

From Figure 8.and Figure 9.,with the increace of velocity of projectile and target, the circuit output signal frequency increases,basically a linear relationship.Compared the Figure 9. and Figure 7., , the output signal frequency and the input frequency satisfy the relation $f^{\prime}=2 v_{\mathrm{r}} f / c$.

From Figure 8,the amplitude of signal changed with the change of projectile and target velocity,the Figure 10. Shows the amplitude relationship.

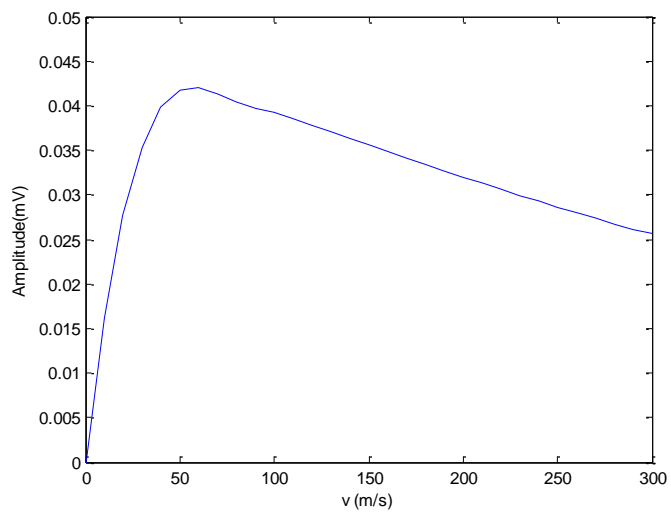

Figure 10.projectile and target velocity amplitude curves

From the Figure 10, the relationship between the velocity and the amplitude is not monotonic. There is an extreme point,when the velocity less than the extreme point,the amplitude of output signal increase with the increase of velocity, when the velocity is more than the extreme point the amplitude of output signal decrease with the increase of velocity.

\section{CONCLUSIONS}

The time domain Doppler effect of UWB fuze is different from the traditional sinusoidal narrow-band fuze,it is demonstrated that because of the time domain Doppler effect,the UWB fuze receiver output frequency is proportional to the frequency of input frequency and the projectile and target velocity.Because of the receiver input signal is the fuze echo signal,the frequency of the echo signal is inversely proportional to the width of the pulse.

In conclusion the output frequency and the input frequency satisfy $f^{\prime}=\frac{2 v_{\mathrm{r}}}{c} f$ UWB receiver is proportional to the projectile target approaching velocity $v_{\mathrm{r}}$ and input frequency, The output signal of the UWB receiver is inverse ratio to the width of fuze transmit pulse signal.

The amplitude of signal changed with the change of projectile and target velocity,the Figure 10. Shows the amplitude relationship.

\section{REFERENCES}

[1] S. Fujita, T. Sakamoto, T. Sato. "2-Dimensional Accurate Imaging with UWB Radar Using Indoor Multipath Echoes for a Target in Shadow Regions,"'[J] IEICE Trans. Commun., 2011,vol. E94-B, pp. 2366-2374

[2] S. Fujita, T. Sakamoto , T. Sato. "Accurate imaging of a moving target in shadow regions with UWB radar using Doppler effect"'J].2012(EuCAP),p 2130-4,2012.

[3] J.D.Kraus.Antenna:For all applications(Third Editions)[M].Beijing, Publishing House Of Electronics Industry,2011.

[4] A.Miessen, Amina,Kupferschmidt, C.Kuchenbecker, HansPeter;Kaiser, Thomas. "Compensation techniques for Doppler effect in UWB-based rotor-telemetry system" 1st Middle East Conference on Antennas and Propagation,2010, MECAP'10.

[5] L.Marrucci. "Spinning the Doppler Effect.[J]"2013,Science 2 August p 464-465.

[6] Kim, Sang-Dong,Lee, Jong-Hun. "Performance analysis of LFMUWB radar based on Doppler frequency" 2010 International Conference on Information and Communication Technology Convergence, ICTC 2010,p 297-298.

[7] V.C.Chen.The Micro -Doppler effect in Radar.[M].Beijing, Publishing House Of Electronics Industry,2013.

[8] P. Meissner, T. Gigl, and K. Witrisal, "UWB Sequential Monte Carlo Positioning using Virtual Anchors " International Conference on Indoor Positioning and Indoor Navigation,2010, pp. 355-364.

[9] M.Gabriella, D.Benedetto,G.Giancola. Understanding Ultra Wide Band Radio Fundamentals[M]. Beijing, Publishing House Of Electronics Industry,2007.

[10] B.Levitas,J.Matuzas,D.Nikita,E.Rafi;N Matan. "UWB Time Domain Radar for moving detection.[J]" 2011 IEEE International Conference on Microwaves, Communications, Antennas and Electronic Systems. 